\title{
Incapacidad laboral en esclerosis múltiple: a propósito de un caso
}

\section{Working disability in multiple sclerosis: a propos of a case}

\section{Gian C. Navarro Chumbes}

Servicio de Prevención de Riesgos Laborales. Hospital Universitario Ramón y Cajal.

Madrid. España.

\section{Marina Fernández Escribano}

Servicio de Prevención de Riesgos Laborales. Hospital Universitario Ramón y Cajal. Madrid. España.

\section{Ignacio Sánchez-Arcilla Conejo}

Servicio de Prevención de Riesgos Laborales. Hospital Universitario Ramón y Cajal.

Madrid. España.

Recibido: 23-11-10

Aceptado: 25-11-10

\section{Correspondencia:}

Gian C. Navarro Chumbes

Servicio de Prevención de Riesgos Laborales.

Hospital Universitario Ramón y Cajal.

Carretera de Colmenar Viejo, Km 9, 100. 28049 Madrid. España.

Correo electrónico: gcnavarro@yahoo.com / gcnavarro@hotmail.com

\section{Resumen}

Enfermera de 34 años de edad que acude a la Unidad Médica de Valoración de Incapacidades del INSS (Instituto Nacional de Seguridad Social) para valoración de Incapacidad Temporal (IT); diagnosticada de Esclerosis Múltiple (EM) hace 10 años, presentó 6 brotes, por lo que requirió tratamiento con Acetato de Glatirámero y se le adaptó el puesto de trabajo. Actualmente tiene un EDSS (Expanded Disability Status Scale) estimado de 1,0, y actualmente no está con tratamiento inmunomodulador.

Este caso clínico lo que busca es conocer de qué manera se valora la incapacidad laboral en los casos de trabajadores que padecen de EM, asimismo cómo se determina el grado de menoscabo en función del EDSS y qué aspectos laborales son tomados en consideración.

Med Segur Trab (Internet) 2010; 56 (221): 323-327

Palabras clave: Esclerosis Múltiple, trabajo, discapacidad, evaluación.

Abstract

A 34-year old woman, nurse, who arrives to the Medical Assessment Disability Unit of SSNI (Social Security National Institute) for to assess Temporary Disability (TD), she was diagnosed of Multiple Sclerosis (MS) 10 years ago, she had 6 outbreaks and because of that she was treated with Glatiramer Acetate and also her workstation was adapted. At the moment she has an EDSS (Expanded Disability Status Scale) of 1.0, and now she does not receive immunomodulator treatment. 
With this case report we want to know the way that labour disability is evaluated for workers that complain of MS, also how the damage degree is determined according to EDSS and what labour aspects are taken into consideration.

Med Segur Trab (Internet) 2010; 56 (221): 323-327

Key words: Multiple Sclerosis, work, disability, evaluation. 


\section{CASO CLÍNICO}

Mujer de 34 años de edad, de profesión enfermera, que acude a la Unidad Médica de Valoración de Incapacidades del INSS para valoración de IT. El motivo de la baja actual es dolor neuropático (no aporta informes).

Fue diagnosticada de EM hace 10 años y ha presentado 6 brotes por lo que requirió tratamiento con Copaxone ${ }^{\circledR}$ (Acetato de Glatirámero). Anteriormente en su hospital trabajaba en la planta de oncología, pero desde hace 3 años, debido a los brotes de EM, fue reubicada en el área de consultas de urología.

Como antecedentes previos fue diagnosticada de fibromialgia, fatiga crónica y cuadro ansioso depresivo.

El tratamiento actual es: Cymbalta ${ }^{\circledR}$ (Hidrocloruro de Duloxetina) $60 \mathrm{mg}$ vía oral (VO) 1-1/2-0, Rivotril ${ }^{\circledR}$ (Clonazepam) $2 \mathrm{mg}$ VO 1-1-1, Lyrica $^{\circledR}$ (Pregabalina) $150 \mathrm{mg}$ VO 1-0-1 1/2, Stilnox ${ }^{\circledR}$ (Zolpidem) $10 \mathrm{mg}$ VO 0-0-1. No está recibiendo tratamiento inmunomodulador.

Al examen físico: funciones superiores normales, colaboradora, no labilidad ni alteración sensoperceptiva, pares craneales normales, fuerza y balance simétricos, no espasticidad clara, reflejos osteotendinosos con hiperreflexia izquierda, no se aprecia clonus en miembros inferiores, babinsky negativo, hemihipoestesia izquierda incluida hemifascies, marcha normal, realiza marcha de puntillas, talones y tándem, pruebas cerebelosas normales, romberg negativo. EDSS estimado de 1,0.

\section{ANTECEDENTES}

La EM es el trastorno inflamatorio más común del sistema nervioso central (SNC) que afecta la mielina tanto del cerebro como de la médula espinal en uno o varios lugares; asimismo es una de las causas más importantes de discapacidad en los adultos jóvenes ${ }^{1}$ que son los que están en actividad laboral. Se sabe que el $50 \%$ de los pacientes evoluciona hacia un déficit persistente a los 15 años de iniciada la enfermedad y el $80 \%$ a los 30 años ${ }^{2,3}$.

La epidemiología de la EM está cambiando en los últimos años y se cuestiona lo tradicionalmente admitido: el gradiente de mayor prevalencia e incidencia norte-sur (en el hemisferio norte) o, lo que es lo mismo, su incremento cuando nos alejamos del ecuador. En la última década existe el consenso mayoritario de un aumento de la prevalencia e incidencia de esta enfermedad en Europa, en la cuenca mediterránea y, posiblemente, en todo el mundo. El aumento de su incidencia puede deberse a una mejor detección de la enfermedad (gracias a la aparición de la resonancia magnética), y el de la prevalencia, a una mayor supervivencia de los pacientes con EM ${ }^{4}$.

Para el diagnóstico de EM se utilizan los criterios de McDonald del año $2005^{5}$, en el que se considera la diseminación de las lesiones desmielinizantes en tiempo y espacio.

Debido a que esta enfermedad afecta a adultos jóvenes que están en actividad laboral es importante valorar la repercusión funcional que tiene la enfermedad sobre las diferentes capacidades de la persona en el ámbito laboral. 


\section{DISCUSIÓN Y CONCLUSIONES}

Al momento de valorar la incapacidad laboral en EM se toman en consideración 3 aspectos:

- Funcionalidad global.

- Funcionalidad dependiente de la actividad laboral (específica del sistema neurológico afectado).

- Tratamientos que está recibiendo el trabajador.

Cabe mencionar que la EDSS de JF Kurtzke ${ }^{6}$ es actualmente la escala más ampliamente utilizada en todo el mundo para valorar el daño o deterioro neurológico, así como la limitación de la actividad en la EM, basado en un sistema de puntuación de ocho sistemas funcionales que nos indica dónde situar el grado de afectación del paciente en una escala de 20 grados de deterioro neurológico con un recorrido entre el 0 y el 10. Los grados del 0 al 3,5 se diferencian en función de las puntuaciones en los sistemas funcionales y a partir del grado 4, los grados se definen en función principalmente de las alteraciones halladas en la marcha que desde el punto de vista laboral es uno de los factores más importantes a tener en cuenta para valorar el puesto de trabajo.

Asimismo en función de la EDSS se puede determinar el grado de menoscabo ${ }^{7}$, de la siguiente manera:

- Ligero: EDSS 0-1,5 (no incapacitante).

- Moderado: EDSS 2-4,5 (incapacidad para trabajos con carga física importante y en relación a las exigencias profesionales).

- Severo: EDSS 5-6,5 (incapacidad para cualquier actividad profesional).

- Muy severo: EDSS > 6,5 (ayuda para las actividades básicas de la vida diaria).

Debemos tener en cuenta que el EDSS nos da una idea global de la funcionalidad del trabajador. Hay lesiones estratégicas del SNC que podrían darnos un grado de menoscabo leve pero que sin embargo son determinantes para el desempeño de un trabajo en concreto, por lo que la evaluación de dicho trabajador sería de incapacidad total de dicho trabajo.

Es importante que un médico del trabajo conozca la relación existente entre la escala EDSS y el grado de menoscabo, y también el considerar algunos aspectos respecto a la EM y el trabajo, como son:

- Se recomienda adaptación del puesto de trabajo según lo requiera.

- Evitar la exposición del trabajador a temperaturas por encima de los $26{ }^{\circ} \mathrm{C}$ ó $27^{\circ} \mathrm{C}$ debido a que producirá un aumento de la temperatura corporal del trabajador, lo cual enlentecería la conducción nerviosa y por consiguiente el agravamiento de los síntomas. Esta medida cumpliría lo establecido en el Anexo III del Real Decreto 486/1997, del 14 de abril, sobre las disposiciones mínimas de seguridad y salud en los lugares de trabajo, en el que se establecen las condiciones ambientales de los lugares de trabajo.

- Para aquellos casos que padezcan epilepsia, el evitar actividades de riesgo.

- Salvo periodos de reagudización no está indicado el reposo laboral.

- Salvo profesionales con importantes requerimientos físicos es deseable una situación de trabajo más que la inactividad prolongada.

- Valorar reducir la carga de trabajo si precisa más tiempo para realizar cada tarea.

- No prolongar la jornada laboral, reduciéndola si es preciso; aunque también podrían reducirse las tareas o alternarlas de modo que las tareas que requieran más actividad física sean realizadas en las primeras horas del día. 
- Mayor número de momentos de descanso.

- El realizar nocturnidad/turnicidad tendría que ser valorado de acuerdo al grado de menoscabo y valorar si está recibiendo algún tipo de tratamiento específico para EM que dificulte su aplicación o manejo en el lugar de trabajo por el hecho de hacer nocturnidad/turnicidad.

Tras el caso clínico podemos concluir que con un EDSS de 1,0 en el que no hay incapacidad, cuyo grado de menoscabo es ligero, y en el que no existe limitación significativa para realizar sus actividades laborales en el puesto adaptado de las consultas de urología, creemos que la trabajadora debe reincorporarse a su puesto de trabajo.

\section{BIBLIOGRAFÍA}

1. Benito-León J, Martin E, Vela L, Villar ME, Felgueroso B, Marrero C, et al. Multiple sclerosis in Móstoles, central Spain. Acta Neurol Scand. 1998; 98: 238-42.

2. Capello E, Voskuhl RR, McFarland HF, Raine CS. Multiple sclerosis: re-expression of a developmental gene in chronic lesions correlates with remyelination. Ann Neurol. 1997; 41:797-805.

3. Atlanta. Georgia. AAN. 115 Anual Meeting. Natural Course of Multiple Sclerosis Redefined: National Inst of Neurological Disorder and Stroke, 16, 1990.

4. Benito-León J, Bermejo-Pareja F. ¿Está cambiando la epidemiología de la esclerosis múltiple? Rev Neurol. 2010; 51 (07):385-386.

5. Polman C, Reingold S, Edan G, et al. Diagnostic criteria for multiple sclerosis: 2005 revisions to the "McDonald Criteria" Ann Neurol. 2005.

6. Kurtzke JF. Rating Neurological impairment in multiple sclerosis: an expanded disability status scale (EDSS). Neurology. 1983; 33: 144452.

7. Autoría múltiple. "Guía de Valoración de Incapacidad Laboral para Médicos de Atención Primaria". Escuela Nacional de Medicina del Trabajo (ENMT). Instituto de Salud Carlos III. Ministerio de Ciencia e Innovación. Madrid. 2009. Álvarez-Blázquez Fernández F, Director. Jardon Dato E, Carbajo Sotillo MD, Terradillos García MJ, Valero Muñoz MR, Robledo Muga F, Maqueda Blasco J, Cortés Barragán R, Veiga de Cabo J, coordinadores.

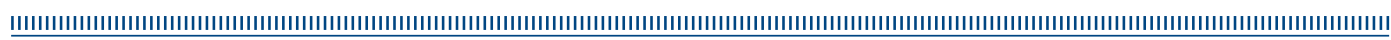

